\title{
Resuscitation with Valproic Acid Alters Inflammatory Genes in a Porcine Model of Combined Traumatic Brain Injury and Hemorrhagic Shock
}

\author{
Ted Bambakidis, Simone E. Dekker,,2 Martin Sillesen,3 Baoling Liu,, Craig N. Johnson, \\ Guang Jin, Helga E. de Vries, Yongqing Li, and Hasan B. Alam ${ }^{1}$
}

\begin{abstract}
Traumatic brain injury and hemorrhagic shock (TBI+HS) elicit a complex inflammatory response that contributes to secondary brain injury. There is currently no proven pharmacologic treatment for TBI+HS, but modulation of the epigenome has been shown to be a promising strategy. The aim of this study was to investigate whether valproic acid (VPA), a histone deacetylase inhibitor, modulates the expression of cerebral inflammatory gene profiles in a large animal model of TBI+HS. Ten Yorkshire swine were subjected to computer-controlled TBI+HS (40\% blood volume). After $2 \mathrm{~h}$ of shock, animals were resuscitated with Hextend (HEX) or HEX+VPA ( $300 \mathrm{mg} / \mathrm{kg}, n=5 / \mathrm{group})$. Six hours after resuscitation, brains were harvested, RNA was isolated, and gene expression profiles were measured using a porcine microarray. Ingenuity Pathway Analysis ${ }^{\circledR}$ (IPA), gene ontology (GO), Parametric Gene Set Enrichment Analysis (PGSEA), and DAVID (Database for Annotation, Visualization, and Integrated Discovery) were used for pathway analysis. Key microarray findings were verified using real-time polymerase chain reaction (PCR). IPA analysis revealed that VPA significantly down-regulated the complement system $(p<0.001)$, natural killer cell communication $(p<0.001)$, and dendritic cell maturation $(p<0.001)$. DAVID analysis indicated that a cluster of inflammatory pathways held the highest rank and gene enrichment score. Real-time PCR data confirmed that VPA significantly down-expressed genes that ultimately regulate nuclear factor-kB (NF-kB)-mediated production of cytokines, such as TYROBP, TREM2, CCR1, and IL-1 $\beta$. This high-throughput analysis of cerebral gene expression shows that addition of VPA to the resuscitation protocol significantly modulates the expression of inflammatory pathways in a clinically realistic model of TBI+HS.
\end{abstract}

Key words: genomics; inflammation; in vivo studies; therapeutic approaches for the treatment of central nervous system injury; traumatic brain injury

\section{Introduction}

$\mathbf{T}$ RAUMATIC BRAIN INJURY (TBI) initiates a complex cascade of cellular and biochemical changes that contribute to dysfunction of neural, glial, and endothelial cells. ${ }^{1}$ Both TBI and hemorrhagic shock (HS) modulate levels of inflammatory mediators, and inflammation may play a key role in the progression of secondary brain injury. This secondary injury includes an acute inflammatory response with blood-brain barrier disruption, edema, activation and infiltration of immune cells, and release of cytokines and chemokines. ${ }^{2-8}$

At present, there are no proven pharmacologic treatment options for TBI. Such therapeutic treatment of TBI, however, has received considerable attention in recent years. ${ }^{9-12}$ For example, a thera- peutic strategy for modulating this neuroinflammatory response may be at the level of the epigenome. One class of promising drugs is histone deacetylase inhibitors (HDACI). Valproic acid (VPA) is routinely used in low doses to manage mood disorders and seizures, but in high doses it has been shown to act as a HDACI.

We have previously shown that VPA creates a prosurvival phenotype via epigenetic modulation in multiple animal models of shock, sepsis, and traumatic injury. ${ }^{13-17}$ For example, the addition of VPA to hetastarch resuscitation significantly decreases brain swelling and lesion size in a swine model of TBI combined with hemorrhagic shock (TBI+HS). ${ }^{14}$

Yet, the precise mechanisms underlying these physiologic changes remain largely unknown. We know that VPA attenuates

\footnotetext{
${ }^{1}$ Department of Surgery, University of Michigan Hospital, Ann Arbor, Michigan.

${ }^{2}$ Department of Anesthesiology, Institute for Cardiovascular Research, VU University Medical Center, Amsterdam, the Netherlands.

${ }^{3}$ Department of Surgical Gastroenterology, Copenhagen University Hospital, Copenhagen, Denmark.

${ }^{4}$ DNA Sequencing Core, University of Michigan, Ann Arbor, Michigan.

${ }^{5}$ Department of Molecular Cell Biology and Immunology, VU University Medical Center, Amsterdam, the Netherlands.
} 
peripheral and central nervous system inflammatory responses by decreasing the production of cytokines and chemokines. ${ }^{13,14,18,19}$ This immunosuppressive effect is also observed in the specific setting of combined TBI+HS. For example, our laboratory has previously demonstrated that administration of VPA attenuates the proinflammatory cytokine response by decreasing the number of CD11b-positive cells as well as myeloperoxidase and IL-1 levels in the brain. ${ }^{14}$ Because combined TBI and HS create tissue damage with a subsequent immune response, the aim of the present study was to determine whether VPA treatment affects the innate and adaptive immune response at the level of gene transcription.

We recently performed a high-throughput RNA microarray analysis on brain tissue from swine subjected to combined TBI+HS insults and resuscitated with the artificial colloid Hextend ${ }^{\circledR}$ (HEX) with or without VPA. The resultant modeling output revealed that more than 1600 probesets were significantly modulated in the HEX+VPA group compared with HEX treatment alone. ${ }^{20}$ Because of the size and complexity of this genomic dataset, we initially needed to restrict our analysis to test the hypothesis that pathways and genes related to cell survival and apoptosis would be significantly different between the groups, which resulted in a previously published study. ${ }^{20}$ For this new study, we used the same treatment groups and array, but employed novel modeling computations and pathway analyses that focused on inflammation. We hypothesized that VPA would decrease brain inflammatory gene expression profiles after TBI+HS.

\section{Methods}

This study was conducted in compliance with the Animal Welfare Act and Federal regulations. The protocol was approved by the Institutional Animal Care and Use Committee and adhered to the principles of the Guide for the Care and Use of Laboratory Animals, Institute for Laboratory Animal Research (1996). Procedures were performed under the direction of an experienced surgeon and supervised by a veterinarian. This animal model of TBI+HS has been described previously in the literature. ${ }^{21}$

In brief, 10 female Yorkshire swine $(42-50 \mathrm{~kg}$, Tufts Veterinary School, Grafton, MA) underwent controlled cortical impact and simultaneous volume-controlled hemorrhage. To expose the dura, a 20-mm burr hole was made on the right side of the skull, next to the coronal and sagittal sutures over the frontal lobe. A TBI of $12 \mathrm{~mm}$ penetration depth was inflicted, and $40 \%$ blood volume was withdrawn through the femoral arterial catheter using a pump.

After the hemorrhage period, animals were kept hypotensive for $2 \mathrm{~h}$ by maintaining the mean arterial pressure (MAP) between 30 and $35 \mathrm{~mm} \mathrm{Hg}$. After $2 \mathrm{~h}$ of hypotension, animals were resuscitated with either (1) HEX (Hospira Inc, Lake Forest, IL) or (2) HEX plus VPA $300 \mathrm{mg} / \mathrm{kg}$ (EMD Biosciences Inc., La Jolla, CA). The volume of HEX resuscitation was equal to the volume of shed blood for each animal. To simulate the pre-hospital use of VPA, this agent was administered $1 \mathrm{~h}$ after hemorrhage and $1 \mathrm{~h}$ before fluid resuscitation at a rate of $100 \mathrm{mg} / \mathrm{kg}$ per hour intravenously. HEX was infused at a rate of $50 \mathrm{~mL} / \mathrm{min}$ in all animals.

\section{Tissue sampling and RNA preparation}

The tissue sampling and RNA preparation methods have been described previously by our laboratory. ${ }^{20}$ Briefly, the brain was harvested after $6 \mathrm{~h}$ of post-resuscitation monitoring. The $30 \mathrm{mg}$ of brain tissue immediately inferior to the primary TBI lesion was homogenized, and RNA was extracted using a RNeasy mini kit (Qiagen, Valencia, CA). The RNA was prepared for microarray hybridization with the GeneChip ${ }^{\circledR}$ WT Plus Reagent Kit (Affymetrix Inc., Santa Clara, CA) according to manufacturer instructions. ${ }^{22}$
Briefly, the RNA was purified and reverse transcribed to synthesize complimentary DNA (cDNA) strands. Amplified RNA was subsequently synthesized, purified, quantitated, and normalized before the second cycle of cDNA synthesis. After RNAse $\mathrm{H}$ digestion of template RNA, the remaining cDNA was purified, quantitated, normalized, and then fragmented and hybridized to the array.

\section{Gene expression analysis}

Gene expression profiles were measured by the University of Michigan DNA Sequencing Core Facility (Ann Arbor, MI) using a Porcine Gene ST 1.1 microarray (Affymetrix, CA; 394,580 probes, 19,212 genes). The gene expression analysis has been described previously by our team. ${ }^{20}$ Briefly, expression values for each gene were calculated using a robust multiarray average, ${ }^{23}$ and microarrayspecific weighted linear models were fitted to the data ${ }^{24}$ to compute the contrasts between HEX and HEX+VPA. Samples were weighted based on a gene-by-gene update algorithm. ${ }^{25}$

Probesets with a fold change of two or greater or below 0.5 with adjusted $p$ values of 0.05 or less were selected. Probesets with a variance over all samples less than 0.1 were not included. ${ }^{26}$ The $p$ values were adjusted for multiple comparisons using false discovery rate (FDR) ${ }^{26}$ Gene expression profiles were analyzed using oligo and limma packages in R. Probesets with more than a twofold change in expression with a FDR-adjusted $p \leq 0.05$ were considered statistically significant.

\section{Pathway analyses}

Inflammation-specific molecular pathways that were associated with altered gene expression in the brain were analyzed between HEX and HEX+VPA groups. Several new network analysis tools were used to test the hypothesis that VPA treatment would attenuate the inflammatory response: Gene Ontology $(\mathrm{GO}),{ }^{27}$ Ingenuity $^{\circledR}$ Pathway Analysis (IPA; Ingenuity Systems, Redwood City, CA), Parametric Gene Set Enrichment Analysis (PGSEA; Broad Institute, Cambridge, MA), ${ }^{28}$ and DAVID (Database for Annotation, Visualization, and Integrated Discovery; v6.7, National Institute of Allergy and Infectious Diseases, National Institutes of Health, Bethesda, MD). ${ }^{29,30}$ Inflammation-specific parameters and annotation terms were input into each pathway analysis tool, which resulted in unique modeling outputs that revealed the effect of VPA on inflammatory gene expression and pathways.

\section{Real-time polymerase chain reaction (PCR)}

Key microarray findings were verified using real-time PCR (Table 1). Briefly, a High-Capacity Reverse Transcription Kit (Applied Biosystems, Carlsbad, CA) was used to convert RNA from each sample into cDNA. A reaction mixture consisting of equal quantities of cDNA, SYBR Green Master Mix, and forward and reverse primers was incubated and analyzed using an ABI PRISM 7300 Real-Time PCR instrument. Porcine-specific primers were custom-made by Real Time Primers (Elkins Park, PA).

Table 1. Selected Genes that Were Analyzed Using Real-Time Polymerase Chain Reaction

Gene name Forward primer $\left(5^{\prime}-3^{\prime}\right)$ Reverse primer $\left(5^{\prime}-3^{\prime}\right)$

CCR1 tgccagaaggtacaggagag gaggtagatggtggtcatgc

IL-1 $\beta \quad$ ctttgaagaagagccatca tgctatcatctcettgcaca

TYROBP caacacatcgctgagacaga gtcetgatttgggetcattt

TREM2 cctcttgaatccagtccaa gggaagtcctctgtttgtgc

CCR1, chemokine receptor 1 ; IL-1 $\beta$, interleukin-1 beta; TYROBP, tyrosine kinase binding protein; TREM2, triggering receptor expressed on myeloid cells 2 . 
Samples were normalized using the difference in critical threshold (ct) between the target gene and actin (delta-ct) and then converted to fold-change. T-tests on PCR data were performed using IBM SPSS Statistics version 20 (IBM, New York).

\section{Results}

IPA

We previously reported an in-depth analysis of genes and pathways related to apoptosis and cell survival from this dataset. ${ }^{20}$ In the present study on inflammation, IPA analysis revealed that the addition of VPA to HEX treatment significantly downregulated the genetic pathway encoding for the complement system $(p<0.001)$, natural killer cell communication $(p<0.001)$, and dendritic cell maturation $(p<0.001)$ (Table 2). Significantly modulated molecules involved in these pathways included toll-like receptor 3 (TLR3), complement component 1 , q subcomponent $\mathrm{B}$ - and C-chains (C1QB and $\mathrm{C} 1 \mathrm{QC}$, respectively), high affinity immunoglobulin epsilon receptor subunit-alpha and -gamma (FCER1A and FCER1G, respectively), as well as tyrosine kinase binding protein (TYROBP) and triggering receptor expressed on myeloid cells 2 (TREM2) $(p<0.05)$.

\section{GO and PGSEA}

$\mathrm{GO}$ analysis indicated that the expression of more than 40 known genes associated with immune system processes were significantly different between the groups ( $p=0.005$, Fig. 1A). PGSEA results revealed significant changes in the expression of genes involved in the immune response (Fig. 1B). Specifically, genes such as interleukin-1 beta (IL-1 beta), TREM2, TYROBP, and chemokine receptor 1 (CCR1) were significantly downexpressed in the HEX+VPA group.

\section{DAVID}

The suite of DAVID tools was used to identify relationships among significantly modulated genes, pathways, and clusters of functionally related pathways. Preliminary analysis using functional annotation clustering on all significantly modulated genes resulted in a group of four GO inflammation annotations as having the highest rank-importance with an enrichment score of 5.51. To better determine the directionality of this enrichment, we reran the DAVID analysis but restricted the gene input list to either significantly up- regulated or downregulated genes. The analysis of downregulated genes revealed a cluster of the same four GO inflammation annotations as in the initial analysis, with a rank-importance of 1 and an increased enrichment score of 8.13 (Table 3).

The DAVID output produced additional inflammation clusters, some of which contained Kyoto Encyclopedia of Genes and Genomes (KEGG) ${ }^{31}$ pathways that showed a downregulation of major immune response pathways. One highly significant pathway was annotated as the systemic lupus erythematosus pathway. Significant mechanisms involved in this pathway include B- and T-cell receptor signaling, antigen processing and presentation, cytokinecytokine receptor interaction, complement and coagulation cascades, and adhesion molecules involved in leukocyte transendothelial migration (Fig. 2).

No inflammation annotation clusters as defined by DAVID were found in the analysis of upregulated genes. We found among others the following annotations being upregulated in the VPA treatment group, however: neuron development and differentiation, synaptic transmission, regulation of neurotransmitter transport and secretion, cell junctions, and negative regulation of apoptosis.

\section{Real-time PCR}

Real-time PCR was used to verify inflammation genes that were identified by the microarray studies as being differentially modulated by VPA. CCR1 was significantly downregulated in the HEX+VPA group compared with HEX alone $(\mathrm{RQ}=0.705, p<0.01)$. Likewise, VPA significantly downregulated the cytokine IL- $1 \beta(\mathrm{RQ}=0.293$, $p<0.01)$. Expression of the membrane proteins TREM2 and TYROBP were both significantly decreased by VPA $(\mathrm{RQ}=0.079$, $\mathrm{RQ}=0.503, p<0.02$ and $p<0.05$, respectively) (Fig. 3).

\section{Discussion}

In this high-throughput analysis of cerebral gene profiling, we investigated whether VPA treatment would affect inflammatory responses after TBI combined with HS. The current study demonstrates that VPA modulates inflammatory genes and genes related to immune regulation, resulting in an overall attenuation of the inflammatory drive. Of interest, pathways involved in wound repair were also induced by VPA, illustrating the broad and protective effect of VPA treatment. A striking reduction of gene

Table 2. Ingenuity Pathway Analysis Output of Inflammatory Pathways with the Greatest Downregulation IN THE HEXTEND+VALPROIC ACID Group

\begin{tabular}{|c|c|c|c|}
\hline Pathway & Ratio & $-\log (\mathrm{p}$ value $)$ & Genes \\
\hline Complement system & 0.185 & 3.47 & CD59, C1QC, C1QB \\
\hline Crosstalk between dendritic cells and natural killer cells & 0.163 & 3.25 & TYROBP, TLR3, TREM2, ACTC1 \\
\hline Dendritic cell maturation & 0.154 & 3.17 & $\begin{array}{l}\text { TYROBP, FCER1G, TLR3, } \\
\text { TREM2, FCGR1A }\end{array}$ \\
\hline Altered $\mathrm{T}$ cell and $\mathrm{B}$ cell signaling in rheumatoid arthritis & 0.118 & 2.27 & SPP1, FCER1G, TLR3 \\
\hline $\begin{array}{l}\text { Role of pattern recognition receptors in recognition } \\
\text { of bacteria and viruses }\end{array}$ & 0.107 & 2.19 & C1RC, C1QB, TLR3 \\
\hline $\begin{array}{l}\text { Fc } \gamma \text { receptor-mediated phagocytosis in macrophages } \\
\text { and monocytes }\end{array}$ & 0.105 & 2.12 & ACTC1, FCGR1A, PLD1 \\
\hline Role of PKR in interferon induction and antiviral response & 0.094 & 1.87 & TLR3, FCGR1A \\
\hline TREM1 signaling & 0.083 & 1.60 & TYROBP, TLR3 \\
\hline $\mathrm{NF}-\kappa \mathrm{B}$ signaling & 0.075 & 1.43 & BMP2, FCER1G, TLR3 \\
\hline Communication between innate and adaptive immune cells & 0.069 & 1.38 & FCER1G, TLR3 \\
\hline
\end{tabular}

Ratio indicates the number of genes in each group that were altered out of the total number of genes in that specific pathway. The $p$ values indicate significance of mapping to the pathway. Genes that were significantly downgregulated are listed for each pathway. 


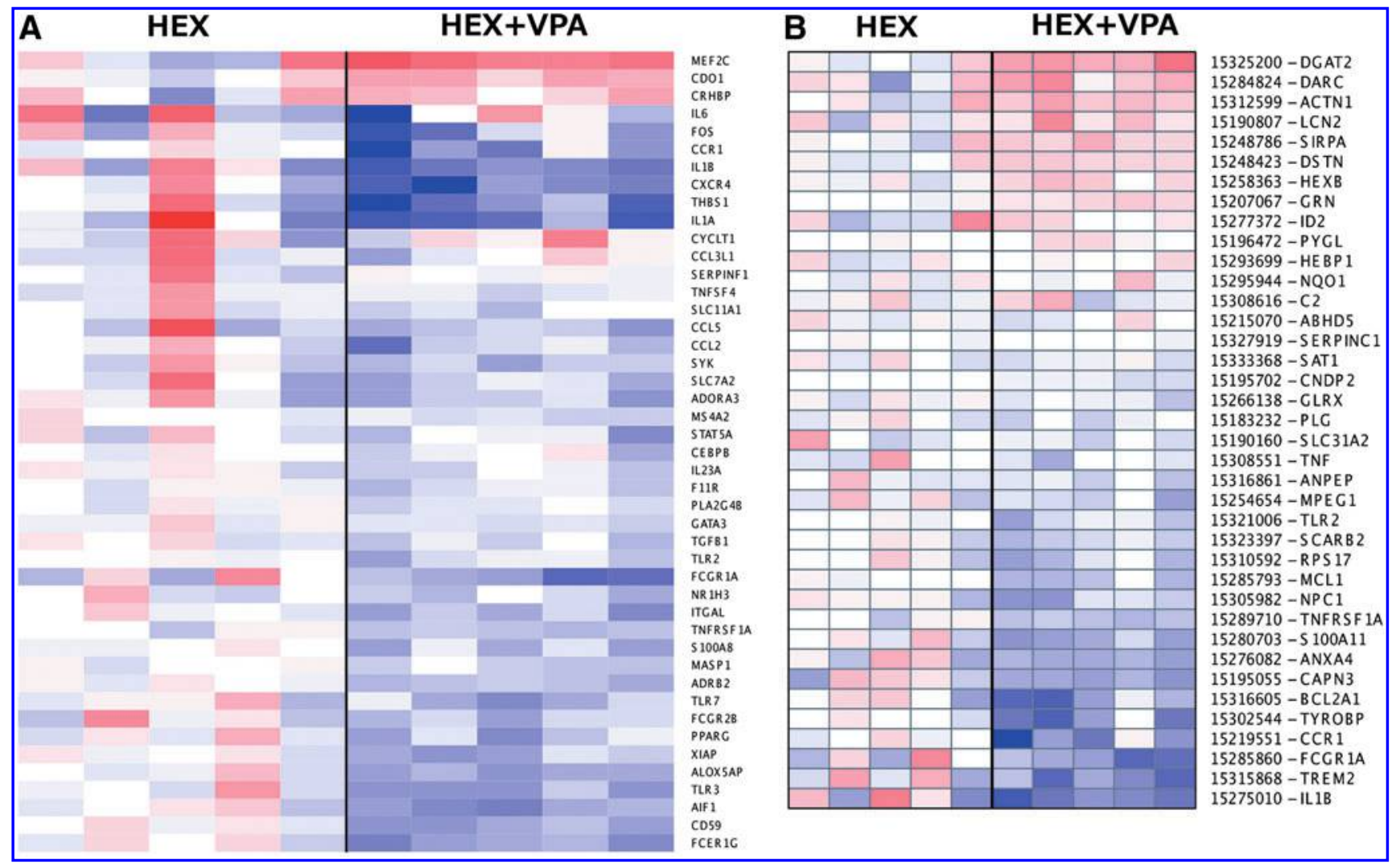

FIG. 1. Heat maps generated from gene ontology and parametric gene set enrichment analysis. (A) Gene ontology heat map based on terms involved in inflammation. A group of nonmodulated genes were omitted for brevity and presentation. (B) Representative PGSEA heat map that was significantly modulated because of valproic acid (VPA). Original annotation data are from Brown and colleagues. ${ }^{39}$ Red, significantly upregulated, and blue, significantly downregulated, for both maps at $p \leq 0.05$. HEX, Hextend. Color image is available online at www.liebertpub.com/neu

expression associated with inflammation was observed. Because inflammation may play a key role in the progression of secondary brain injury and associated edema, our findings may in part explain why VPA treatment is associated with a decrease in brain lesion size and brain swelling after combined TBI and HS. ${ }^{14}$
Several studies have described the benefits of VPA treatment and its role in attenuating inflammatory responses. ${ }^{13,14,18,19,32}$ This is the first study, however, that investigates a number of the underlying genes responsible for this effect. Our study uses several lines of evidence to demonstrate that VPA treatment attenuates the

Table 3. Database for Annotation, Visualization, and Integrated Discovery Functional Annotation Clustering Results from Downregulated Gene List in the Hextend+Valproic Acid Group (Enrichment Score 8.13)

\begin{tabular}{|c|c|c|c|c|c|}
\hline Term & Percent & $\begin{array}{c}\text { Fold } \\
\text { Enrichment }\end{array}$ & $\mathrm{P}$ & $F D R$ & Genes \\
\hline $\begin{array}{l}\text { GO:0009611 } \\
\text { Response to } \\
\text { wounding }\end{array}$ & 21.6 & 6.38 & $4.26 \times 10^{-10}$ & $6.98 \times 10^{-7}$ & $\begin{array}{l}\text { BMP2, GATM, AIF1, CCR1, TLR3, C1QC, CD180, } \\
\text { S100A12, C1QB, CXCR4, CD59, IL1B, VCAN, VSIG4, } \\
\text { THBS1, SELE, PLAU, IL1A, SPP1 }\end{array}$ \\
\hline $\begin{array}{l}\text { GO:0006952 } \\
\text { Defense } \\
\text { response }\end{array}$ & 22.7 & 5.79 & $6.26 \times 10^{-10}$ & $1.03 \times 10^{-6}$ & $\begin{array}{l}\text { BMP2, PLD1, NCF2, AIF1, CCR1, TLR3, C1QC, CD180, } \\
\text { S100A12, C1QB, CXCR4, FCGR1A, IL1B, VSIG4, } \\
\text { THBS1, MX2, SELE, IL1A, SPP1, TYROBP }\end{array}$ \\
\hline $\begin{array}{l}\text { GO:0006954 } \\
\text { Inflammatory } \\
\text { response }\end{array}$ & 17.0 & 8.22 & $2.41 \times 10^{-9}$ & $3.96 \times 10^{-6}$ & $\begin{array}{l}\text { BMP2, AIF1, CCR1, TLR3, C1QC, CD180, S100A12, C1QB, } \\
\text { CXCR4, IL1B, VSIG4, THBS1, SELE, IL1A, SPP1 }\end{array}$ \\
\hline $\begin{array}{l}\text { GO:0006955 } \\
\text { Immune } \\
\text { response }\end{array}$ & 18.2 & 4.13 & $4.62 \times 10^{-6}$ & $7.58 \times 10^{-3}$ & $\begin{array}{l}\text { AQP9, NCF2, CCR1, TLR3, C1QC, CD180, PDCD1LG2, } \\
\text { C1QB, CXCR4, FCGR1A, IL1B, FCER1G, VSIG4, } \\
\text { TREM2, THBS1, IL1A }\end{array}$ \\
\hline
\end{tabular}

Percent represents the number of genes involved of the total number of genes in the input gene list. Fold enrichment is the magnitude of enrichment relative to the normal expression in the genome. The $p$ value indicates the significance of gene-term enrichment of the annotation term groups. Calculation is based on a modified Fisher exact test (EASE score), with smaller values indicating greater significance. FDR (false discovery rate) is a testing correction technique that globally corrects enrichment $p$ values to control family-wide FDR $\leq 0.05$. GO, gene ontology. 


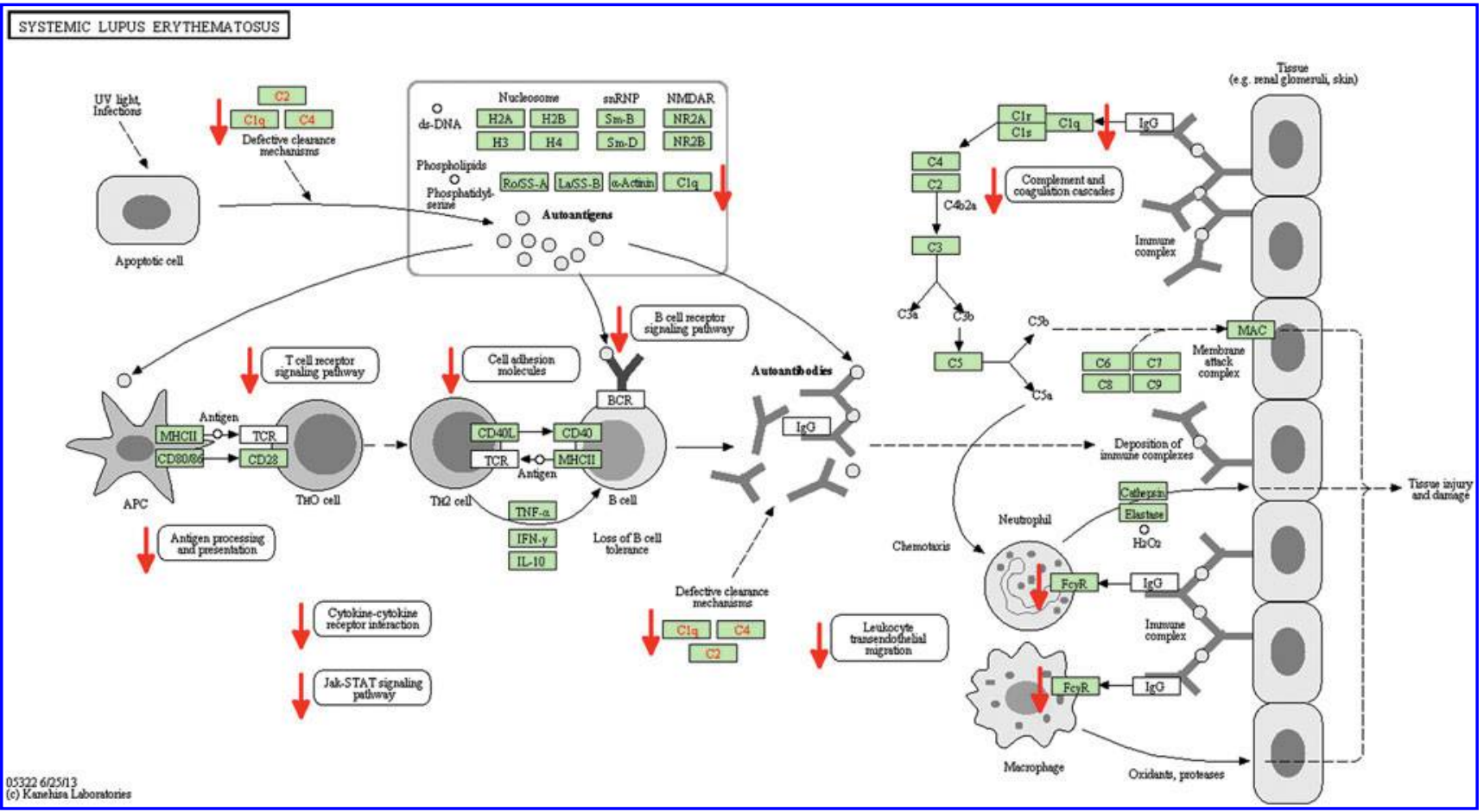

FIG. 2. Kyoto Encyclopedia of Genes and Genomes (KEGG) inflammatory pathway describing modulation of gene expression from the underlying dataset of downregulated genes. The original pathway is annotated for systemic lupus erythematosus (KEGG HSA05322). Red arrows have replaced KEGG asterisks for clarity of presentation. Color image is available online at www.liebertpub.com/neu

transcription of genes related to both innate and adaptive immune responses. For example, GO analysis showed that the expression of more than 40 known genes associated with the immune system were significantly different between the HEX and HEX-VPA groups. Likewise, PGSEA results showed significant changes in the expression of genes involved in the immune response (Fig. 1). Some of the genes that were significantly downregulated in the VPA group were, among others, IL-1 beta, TREM2, TYROBP, and CCR1. IPA results supported the GO and PGSEA datasets by demonstrating that VPA significantly downregulated the innate and

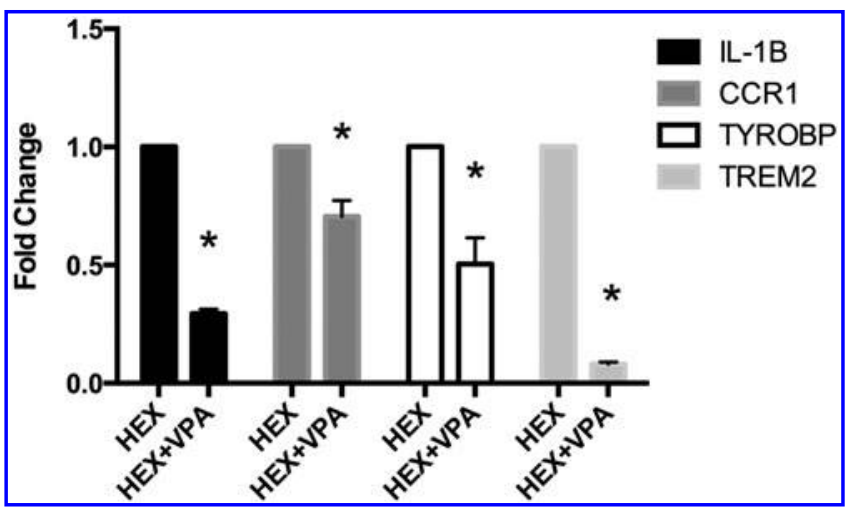

FIG. 3. Real-time polymerase chain reaction (PCR) data showing changes in inflammatory gene expression. Hextend + valproic acid (HEX+VPA) expression was computed relative to HEX alone, which served as the control. Data are shown as mean \pm standard error of the mean. IL-1 $\beta$, interleukin 1-beta; CCR1, chemokine receptor 1; TREM2, triggering receptor expressed on myeloid cells 2; TYROBP, tyrosine kinase binding protein. $* p \leq 0.05$ compared with HEX. adaptive immune responses (Table 2). Not surprisingly, TYROBP and TREM2 are key players in these pathways as well.

A suite of related genes were common among the pathway analyses. While unknown for their roles in trauma and shock, both TREM2 and TYROBP (alternatively referred to as DAP12 or KARAP) serve important roles in inflammation. These cell surface receptors comprise a signaling complex that has been found in a variety of cells involved in the immune response, such as granulocytes, macrophages, and microglia. ${ }^{33-37}$ Expression and activation of the TYROBP-TREM2 complex may be intrinsic or extrinsic. Briefly, rapid TREM2 translocation to the cell surface has been reported because of cytokine-mediated activation in macrophages $^{38}$ and on microglia after an infiltration of macrophages into the central nervous system. ${ }^{40}$ The extrinsic pathway requires activation via a wide variety of ligands that are still poorly described. ${ }^{34}$ Ligand binding to TREM2 stimulates phosphorylation of TYROBP, which in turn activates a variety of downstream cellular pathways, such as the phosphatidylinositol 3-kinase-Akt pathway. TYROBP activation of zeta-chain-associated protein kinase 70 (ZAP70) causes the phosphorylation of Akt, which in turn may regulate nuclear factor-kB (NF-kB)-mediated production of cytokines. ${ }^{34}$ In addition, activation of ZAP70 kinase regulates NF-kB and mitogen-activated protein kinase activity via the second messenger diacylglycerol. ${ }^{34,41-43}$ This effect on the Akt pathway supports our previous work, which showed that VPA treatment activates the Akt pathway in a rodent model of HS. ${ }^{44}$

CCR 1 and IL- $1 \beta$ are also known to direct the activity of NF-kB. CCR1 is a chemokine receptor on macrophages and lymphocytes that has been shown to regulate NF-kB. ${ }^{45}$ Similarly, IL- $1 \beta$ is a well known proinflammatory cytokine that induces the transcription of other cytokines via NF-kB regulation. ${ }^{46}$ Collectively, CCR1, IL$1 \beta$, and the TYROBP-TREM2 complex each regulate NF-kB 
activity and the subsequent production of proinflammatory genes. Although some reports suggest that TYROBP-TREM2 may be involved in the inhibition of the immune response, ${ }^{47,48}$ our modeling and validation data suggest that decreased expression of this complex, as well as CCR1 and IL- $1 \beta$, may have played a key role in attenuating the inflammatory response. This is a unique new finding, because the effect of VPA on this pathway has yet to be described in the literature.

DAVID analysis revealed a downregulation of functionally related inflammation pathways with an enrichment score of 8.13 (Table 3), as well as significantly decreased expression of major immune response pathways such as the KEGG lupus inflammation pathway, which includes numerous genes associated with immune regulation (Fig. 2). Yet, the DAVID modeling output revealed several other alterations in gene expression that may work in tandem with inflammation. For example, several nervous system pathways involving neuronal development and differentiation, synaptic transmission, and neurotransmitter regulation showed an increase in expression from VPA. Thus, upregulation of these neuroprotective pathways may also in part underlie the observed beneficial effects of VPA. Together these data highlight that VPA is a potentially promising new treatment strategy for TBI and HS, not only because of its anti-inflammatory effects but also because of its neuroprotective properties.

Our data illustrate that combined TBI and HS induce a profound inflammatory response, and subsequent inflammation may play a key role in the progression of secondary brain injury and associated edema. The exact mechanism of how the interplay of TBI and HS affect the inflammatory response remains unclear, however. HS and TBI may exert different and even opposing effects on the immune system. A recent study described the inflammatory response when adding HS to a murine model of TBI. ${ }^{49}$ TBI alone elicited a proinflammatory cytokine response, but the addition of HS shifted the cytokine profile to a more anti-inflammatory state. This reduction in proinflammatory response was associated with increased IL-10 serum levels in these animals, suggesting that neuroinflammation may also exert beneficial effects after trauma. For example, inflammation may positively influence the outcome in the more long-term, chronic post-injury recovery period. Scherbel and associates $^{49}$ demonstrated that TNF-alpha may be deleterious in the acute post-TBI period, whereas this cytokine may facilitate longterm behavioral recovery and histological repair after neurotrauma. ${ }^{50}$ Interestingly, the brain has been shown to regionally alter TNF mRNA gene expression. ${ }^{51}$ Moreover, Sinz and colleagues ${ }^{52}$ suggested that inducible nitric oxide synthethase (iNOS), which is induced in both secondary damage and in recovery from brain injury, may have a positive effect on long-term outcome after TBI.

Yet, the time course of inflammation after injury must be considered. Acute phase inflammation in the hours immediately after injury generates rapid lesion formation and edema. In the confined volume of the skull, such changes in intracranial pressure may have severe and even deadly consequences. In contrast, chronic, long-term inflammation may be beneficial by attenuating edema, clearing cellular debris by activated microglia and infiltrated macrophages, and promoting fibrosis and scar formation of the lesion in the longer term.

In the present study, the changes in gene expression were measured $8 \mathrm{~h}$ after the TBI insult ( $6 \mathrm{~h}$ after treatment). Because of this short time frame, it is likely that we only captured the acute phase of the inflammatory response to TBI+HS. In this context, our observation that VPA-treated animals experienced a decrease in inflammatory gene expression may suggest that VPA is attenuating excessive neuroinflammation. Moreover, VPA induced the expression of genes involved in wound repair and tissue remodeling. Because VPA resuscitation was associated with decreased brain lesion size and improved neurologic recovery, ${ }^{14}$ we speculate that this attenuation of the inflammatory response may be one of the possible positive effects of VPA.

Our group and others have demonstrated that the choice of resuscitation fluid plays an important role in the severity of secondary brain injury. ${ }^{21,53,54}$ Compared with crystalloids, artificial colloids (e.g. HEX), and fresh frozen plasma (FFP) have shown an increased ability to limit the extent of brain lesion size and swelling. ${ }^{21,53}$ While fluids do influence gene expression, such effects are likely minor compared with epigenetic modulators such as VPA, a known HDACI.

Accordingly, the goal of this study was to describe the specific changes after VPA treatment. Because of the severity of this model, however, fluid resuscitation was necessary to increase the likelihood of animal survival to the $6 \mathrm{~h}$ post-resuscitation ( $8 \mathrm{~h}$ post-TBI) time of sacrifice. Our choice of HEX reflects the United States military's interest in this resuscitation fluid. First, as an artificial colloid, HEX possesses a volume-sparing effect relative to physiologic saline. Second, the military is interested in battlefield-ready, shelf-stable resuscitation fluids, which would make blood products such as FFP logistically unfeasible. We should note that VPA-induced changes in gene expression are likely to differ when given in combination with other crystalloids, colloids, or blood products. Such different combinations may serve as an important focus of future research.

While this study suggests that VPA may modulate some pathways involved in the inflammatory response, it did have several important limitations. One major limitation of this study is the lack of controls to clearly elucidate both the consequences of the insults as well as the effect of VPA on inflammatory gene expression. As indicated earlier, hemorrhage may evoke complex effects on brain and systemic inflammation after TBI, which may lead to an attenuated neuroinflammatory response compared with TBI alone. ${ }^{48}$ Incorporating additional control and sham groups (e.g., naive, anesthesia, HS alone, TBI alone) could improve our understanding of neuroinflammation and the effect of VPA. Financial and logistical limitations, ethical considerations, as well as the tremendous size and complexity of genomic studies, however, necessarily restricted our ability to include additional groups. As such, our results should be considered as exploratory and descriptive, rather than a definitive investigation of neuroinflammation and VPA after TBI+HS. Moreover, many variables in the model are likely to alter gene expression, including anesthesia, instrumentation, shock, TBI, and resuscitation. We therefore controlled for these variables by using VPA administration as our only independent variable.

Further, this study only focused on the effects of VPA on inflammatory responses after TBI and HS insults. We chose to focus on genes that were common between GO, IPA, PGSEA, and DAVID, such as TYROBP, TREM2, CCR1, and IL- $1 \beta$. It is very likely, however, that numerous other inflammatory and noninflammatory mechanisms play a role in explaining the benefits of VPA treatment as well. Moreover, our study did not elucidate the relationship between alterations in gene expression, as measured by RNA expression, and protein levels and functional outcome. Our lab is currently conducting TBI+HS long-term survival experiments to fill this gap between modulation of gene expression and its translation to chronic neuroinflammatory processes and functional outcome.

Finally, a shortcoming of this study is the poor annotation of the porcine genome relative to human and rodent. While the expression 
of more than 1600 genes was altered by VPA, only a fraction of these have been completely described. Selecting a different model organism with a completely annotated genome could improve our understanding of the beneficial effects of VPA, yet it may sacrifice the considerable clinical realism of this model.

\section{Conclusion}

This is the first high-throughput analysis of cerebral inflammatory gene profiling after TBI+HS. We found that VPA modulates genes and pathways related to inflammation. This modulation of the inflammatory response after TBI+HS may partly explain the reduction in brain lesion size and brain swelling that we observed in VPA treated animals. VPA may serve as a promising pharmacologic agent for the management of combined TBI+HS.

\section{Acknowledgments}

This article was presented at the 10th Annual Academic Surgical Congress (ASC), February 3-5, 2015, Las Vegas, Nevada. This study was funded by a grant from the US Army Medical Research Material Command GRANTT00521959 (to HBA). We would also like to acknowledge generous support for translational research in traumatic brain injury by the Joyce and Don Massey Foundation.

\section{Author Disclosure Statement}

No competing financial interests exist.

\section{References}

1. Graham, D.I., McIntosh, T.K., Maxwell, W.L., and Nicoll, J.A (2000). Recent advances in neurotrauma, J. Neuropathol. Exp. Neurol. 59, 641-651.

2. Soares, H.D., Hicks, R.R., Smith, D., and McIntosh, T.K. (1995). Inflammatory leukocytic recruitment and diffuse neuronal degeneration are separate pathological processes resulting frm traumatic brain injury, J. Neurosci. 15, 8223-8233.

3. Shohami, E., Novikov, M., Bass, R., Yamin, A., and Gallily, R. (1994). Closed head injury triggers early production of TNFa and IL-6 by brain tissue, J. Cereb. Blood Flow Metab. 14, 615-619.

4. Holmin, S., Soderlund, J., Biberfeld, P., and Mathiesen, T. (1998). Intracerebral inflammation after human brain contusion, Neurosurgery 42, 291-299.

5. Stahel, P.F., Shohami, E., Younis, F.M., Kariya, K., Otto, V.I., Lenzlinger, P.M., Grosjean, M.B., Eugster, H.P., Trentz, O., Kossmann, T., and Morganti-Kossmann, M.C. (2000). Experimental closed head injury: analysis of neurological outcome, blood-brain barrier dysfunction, intracranial neutrophil infiltration, and neuronal cell death in mice deficient in genes for proinflammatory cytokines, J. Cereb. Blood Flow Metab. 20, 369-380.

6. Lenzlinger, P.M., Morganti-Kossmann, M.C., Laurer, H.L., and T.K. McIntosh, T.K. (2001). The duality of the inflammatory response to traumatic brain injury, Mol. Neurobiol. 24, 169-181.

7. Whalen, M.J.,Carlos, T.M., Kochanek, P.M., Clark, R.S., Heineman, S., Schiding, J.K., Franicola, D., Memarzadeh, F., Lo, W., Marion, D.W., and DeKosky, S.T. (1999). Neutrophils do not mediate bloodbrain barrier permeability early after controlled cortical impact in rats, J. Neurotrauma 16, 583-594.

8. Morganti-Kossmann, M.C., Rancan, M., Stahel, P.F., and T. Kossmann, T. (2002). Inflammatory response in acute traumatic brain injury: a double-edged sword, Curr. Opin. Crit Care 8, 101-105

9. Luer, M.S., Rhoney, D.H., Hughes, M., and Hatton, J. (1996). New pharmacologic strategies for acute neuronal injury. Pharmacotherapy $16,830-848$.

10. McIntosh, T.K., Juhler, M., and Wieloch, T. (1998). Novel pharmacologic strategies in the treatment of experimental traumatic brain injury: 1998. J. Neurotrauma 15, 731-769.
11. Laurer H.L., and McIntosh T.K. (2001). Pharmacologic therapy in traumatic brain injury: update on experimental treatment strategies. Curr. Pharm. Des. 7, 1505-1516.

12. Han, Z., Lat, I., and Pollard, S.R. (2014). Safety and efficacy of corticosteroid use in neurologic trauma. J. Pharm. Pract. 27, 487-495.

13. Kim, K., Li, Y., Jin, G., Chong, W., Liu, B., Lu, J., Lee, K., Demoya, M., Velmahos, G., and Alam, H.B. (2012). Effect of valproic acid on acute lung injury in a rodent model of intestinal ischemia reperfusion. Resuscitation 83, 243-248.

14. Jin, G., Duggan, M., Imam, A., Demoya, M.A., Sillesen, M., Hwabejire, J., Jepsen, C.H., Liu, B., Mejaddam, A.Y., Lu, J., Smith, W.M., Velmahos, G.C., Socrate, S., and Alam, H.B. (2012). Pharmacologic resuscitation for hemorrhagic shock combined with traumatic brain injury. J. Trauma Acute Care Surg. 73, 1461-1470.

15. Liu, Z., Li, Y., Chong, W., Deperalta, D.K., Duan, X., Liu, B., Halaweish, I., Zhou, P., and Alam, H.B. (2014). Creating a prosurvival phenotype through a histone deacetylase inhibitor in a lethal two-hit model. Shock 41, 104-108.

16. Fukudome E.Y., Kochanek A.R., Li Y., Smith E.J., Liu B., Kheirbek T., Lu J., Kim K., Hamwi, K., Velmahos, G.C., and Alam, H.B. (2010). Pharmacologic resuscitation promotes survival and attenuates hemorrhage-induced activation of extracellular signal-regulated kinase 1/2. J. Surg. Res. 163, 118-126.

17. Fukudome, E.Y., Li, Y., Kochanek, A.R., Lu, J., Smith, E.J., Liu, B., Kim, K., Velmahos, G.C., deMoya, M.A., and Alam, H.B. (2012). Pharmacologic resuscitation decreases circulating cytokine-induced neutrophil chemoattactant-1 levels and attenuates hemorrhageinduced acute lung injury. Surgery 152, 254-261.

18. Ichiyama, T., Okada, K., Lipton, J.M., Matsubara, T., Hayashi, T., and Furukawa, S. (2000). Sodium valproate inhibits production of TNFalpha and IL-6 and activation of NF-kappaB. Brain Res. 857, 246-251.

19. Zhang, Z., Zhang, Z.Y., Fauser, U., and Schluesener, H.J. (2008). Valproic acid attenuates inflammation in experimental autoimmune neuritis. Cell Mol. Life Sci. 65, 4055-4065.

20. Dekker, S.E., Bambakidis, T., Sillesen, M., Liu, B., Johnson, C.N., Jin, G., Li, Y., and Alam, H.B. (2014). Effect of pharmacologic resuscitation on the brain gene expression profiles in a swine model of traumatic brain injury and hemorrhage. J. Trauma Acute Care Surg. 77, 906-912.

21. Jin, G., deMoya, M.A., Duggan, M., Knightly, T., Mejaddam, A.Y., Hwabejire, J., Lu, J., Smith, W.M., Kasotakis, G., Velmahos, G.C., Socrate, S., and Alam, H.B. (2012). Traumatic brain injury and hemorrhagic shock: evaluation of different resuscitation strategies in a large animal model of combined insults. Shock 38, 49-56.

22. Affymetrix: GeneChip ${ }^{\circledR} 3^{\prime}$ IVT Express Kit, User Manual. P/N 702646 Rev. 5. 2008-2009. Available at: http://www.umich.edu/ caparray/Files/protocols/affymetrix/3_ivt_express_kit_manual.pdf Accessed: December 6, 2014.

23. Irizarry, R.A., Hobbs, B., Collin, F., Beazer-Barclay, Y.D., Antonellis, K.J., Scherf, U., and Speed, T.P. (2003). Exploration, normalization, and summaries of high density oligonucleotide array probe level data. Biostatistics 4, 249-264.

24. Smyth, G.K. (2004). Linear models and empirical bayes methods for assessing differential expression in microarray experiments. Stat. Appl. Genet. Mol. Biol. 3, 1-25.

25. Ritchie, M.E., Diyagama, D., Neilson, J., van Laar, R., Dobrovic, A., Holloway, A., and Smyth, G.K. (2006). Empirical array quality weights in the analysis of microarray data. BMC Bioinformatics 7, 261-277.

26. Benjamini, Y., and Hochberg, Y. (1995). Controlling the false discovery rate: a practical and powerful approach to multiple testing. J. R. Statist. Soc. B. 57, 289-300.

27. Ashburner, M., Ball, C.A., Blake, J.A., Botstein, D., Butler, H., Cherry, J.M., Davis, A.P., Dolinski, K., Dwight, S.S., Eppig, J.T., Harris, M.A., Hill, D.P., Issel-Tarver, L., Kasarskis, A., Lewis, S., Matese, J.C., Richardson, J.E., Ringwald, M., Rubin, G.M., and Sherlock G. (2000). Gene ontology: tool for the unification of biology. The Gene Ontology Consortium. Nat. Genet. 25, 25-29.

28. Subramanian, A., Tamayo, P., Mootha, V.K., Mukherjee, S., Ebert, B.L., Gillette, M.A., Paulovich, A., Pomeroy, S.L., Golub, T.R., Lander, E.S., and Mesirov, J.P. (2005). Gene set enrichment analysis: a knowledge-based approach for interpreting genome-wide expression profiles. Proc. Natl. Acad. Sci. U. S. A. 102, 15545-15550.

29. Huang, da W., Sherman, B.T., and Lempicki, R.A. (2009). Systematic and integrative analysis of large gene lists using DAVID bioinformatics resources. Nature Protoc. 4, 44-57. 
30. Huang, D.W., Sherman, B.T., and Lempicki, R.A. (2009). Bioinformatics enrichment tools: paths toward the comprehensive functional analysis of large gene lists. Nucleic Acids Res. 37, 1-13.

31. Kanehisa M., and Goto S. (2000). KEGG: Kyoto Encyclopedia of Genes and Genomes. Nucleic Acids Res. 28, 27-30.

32. Li, Y., and Alam, H.B. (2012). Creating a pro-survival and antiinflammatory phenotype by modulation of acetylation in models of hemorrhagic and septic shock. Adv. Exp. Med. Biol. 710, 107-133.

33. Ma, J., Jiang, T., Tan, L., and Yu, J.T. (2015). TYROBP in Alzheimer's disease. Mol. Neurobiol. 51, 820-826.

34. Paradowska-Gorycka, A., and Jurkowska, M. (2013). Structure, expression pattern and biological activity of molecular complex TREM2/DAP12. Hum. Immunol. 74, 730-737.

35. Tomasello, E., and Vivier, E. (2005). KARAP/DAP12/TYROBP: three names and a multiplicity of biological functions. Eur. J. Immunol. 35, 1670-1677.

36. Kiialainen, A., Hovanes, K., Paloneva, J., Kopra, O., and Peltonen, L. (2005). Dap12 and Trem2, molecules involved in innate immunity and neurodegeneration, are co-expressed in the CNS. Neurobiol. Dis. 18, 314-322.

37. Bouchon, A., Hernandez-Munain, C., Cella., M., and Colonna, M. (2001). A DAP12-mediated pathway regulates expression of CC chemokine receptor 7 and maturation of human dendritic cells. J. Exp. Med. 194, 1111-1122.

38. Turnbull, I.R., Gilfillan, S., Cella, M., Aoshi, T., Miller, M., Piccio, L., Hernandez, M., and Colonna, M. (2006). Cutting edge: TREM-2 attenuates macrophage activation. J. Immunol. 177, 3520-3524.

39. Brown, A.L., Wilkinson, C.R., Waterman, S.R., Kok, C.H., Salerno, D.G., Diakiw, S.M., Reynolds, B., Scott, H.S., Tsykin, A., Glonek, G.F., Goodall, G.J., Solomon, P.J., Gonda, T.J., and D'Andrea, R.J. (2006). Genetic regulators of myelopoiesis and leukemic signaling identified by gene profiling and linear modeling. J. Leukoc. Biol. 80, 433-447.

40. Piccio, L., Buonsanti. C., Mariani, M., Cella, M., Gilfillan. S., and Cross, A.H., Colonna, M., and Panina-Bordignon, P. (2007). Blockade of TREM-2 exacerbates experimental autoimmune encephalomyelitis. Eur. J. Immunol. 37, 1290-1301.

41. Smith-Garvin, J.E., Koretzky, G.A., and Jordan, M.S. (2009). T cell activation. Annu. Rev. Immunol. 27, 591-619.

42. Abraham, R.T., and Weiss, A. (2004). Jurkat T cells and development of the T-cell receptor signalling paradigm. Nat. Rev. Immunol. 4, 301-308.

43. Okkenhaug, K., Ali, K., and Vanhaesebroeck, B. (2007). Antigen receptor signalling: a distinctive role for the p110delta isoform of PI3K. Trends Immunol. 28, 80-87.

44. Bambakidis, T., Dekker, S.E., Liu, B., Maxwell, J., Chtraklin, K., Linzel, D., Li, Y., and Alam, H.B. (2015). Hypothermia and valproic acid activate prosurvival pathways after hemorrhage. J. Surg. Res. 196, 159-165.

45. Lee, M.M., and Wong, Y.H. (2009). CCR1-mediated activation of nuclear factor-kB in THP-1 monocytic cells involves pertussis toxininsensitive Galpha14 and Galpha16 signaling cascades. J. Leukocyte Biol. 86, 1319-1329.
46. Wesche, H., Korherr, C., Kracht, M., Falk, W., Resch, K., and Martin, M.U. (1997). The interleukin-1 receptor accessory protein (IL-1RAcP) is essential for IL-1-induced activation of interleukin-1 receptorassociated kinase (IRAK) and stress-activated protein kinases (SAP kinases). J. Biol. Chem. 272, 7727-7731.

47. Helming, L., Tomasello, E., Kyriakides, T.R., Martinez, F.O., Taki, T., Gordon, S., and Vivier, E. (2008). Essential role of DAP12 signaling in macrophage programming into a fusion-competent state. Sci. Signal 1, ra11.

48. Hamerman, J.A., Tchao, N.K., Lowell, C.A., and Lanier, L.L. (2005) Enhanced toll-like receptor responses in the absence of signaling adaptor DAP12. Nat. Immunol. 6, 579-586.

49. Shein, S.L., Shellington, D.K., Exo, J.L., Jackson, T.C., Wisniewski, S.R., Jackson, E.K., Vagni, V.A., Bayir, H., Clark, R.S., Dixon, C.E., Janesko-Feldman, K.L., and Kochanek, P.M. (2014). Hemorrhagic shock shifts the serum cytokine profile from pro- to anti-inflammatory after experimental traumatic brain injury in mice. J. Neurotrauma 31 , $1386-1395$.

50. Scherbel, U., Raghupathi, R., Nakamura, M., Saatman, K.E., Trojanowski, J.Q., Neugebauer, E., Marino, M.W., and McIntosh, T.K. (1999). Differential acute and chronic responses of tumor necrosis factor-deficient mice to experimental brain injury. Proc. Natl. Acad. Sci. U. S. A. 96, 8721-8726.

51. Fan, L., Young, P.R., Barone, F.C., Feuerstein, G.Z., Smith, D.H., and McIntosh, T.K. (1996). Experimental brain injury induces differential expression of tumor necrosis factor-alpha mRNA in the CNS. Brain Res. Mol. Brain Res. 36, 287-291.

52. Sinz, E.H., Kochanek, P.M., Dixon, C.E., Clark, R.S., Carcillo, J.A., Schiding, J.K., Chen, M., Wisniewski, S.R., Carlos, T.M., Williams, D., DeKosky, S.T., Watkins, S.C., Marion, D.W., and Billiar, T.R. (1999). Inducible nitric oxide synthase is an endogenous neuroprotectant after traumatic brain injury in rats and mice. J. Clin. Invest. $104,647-656$

53. Imam, A., Jin, G., Sillesen, M., Dekker, S.E., Bambakidis, T., Hwabejire, J.O., Jepsen, C.H., Halaweish, I., and Alam, H.B. (2015). Fresh frozen plasma resuscitation provides neuroprotection compared to normal saline in a large animal model of traumatic brain injury and polytrauma. J. Neurotrauma 32, 307-313.

54. Dekker, S.E., Sillesen, M., Bambakidis, T., Jin, G., Liu, B., Boer, C., Johansson, P.I., Halaweish, I., Maxwell, J., and Alam, H.B. (2014). Normal saline influences coagulation and endothelial function after traumatic brain injury and hemorrhagic shock in pigs. Surgery 156, 556-563.

Address correspondence to:

Hasan B. Alam, MD

Department of General Surgery University of Michigan Hospital 1500 E. Medical Center Drive Ann Arbor, MI 48109

E-mail: alamh@med.umich.edu 


\section{This article has been cited by:}

1. Vahagn C. Nikolian, Patrick E. Georgoff, Manjunath P. Pai, Isabel S. Dennahy, Kiril Chtraklin, Hassan Eidy, Mohamed H. Ghandour, Yanyan Han, Ashok Srinivasan, Yongqing Li, Hasan B. Alam. 2017. Valproic acid decreases brain lesion size and improves neurologic recovery in swine subjected to traumatic brain injury, hemorrhagic shock, and polytrauma. Journal of Trauma and Acute Care Surgery 83:6, 1066-1073. [Crossref]

2. G. Fond, N. Resseguier, F. Schürhoff, O. Godin, M. Andrianarisoa, L. Brunel, E. Bulzacka, B. Aouizerate, F. Berna, D. Capdevielle, I. Chereau, T. D’Amato, C. Dubertret, J. Dubreucq, C. Faget, F. Gabayet, C. Lançon, P. M. Llorca, J. Mallet, D. Misdrahi, C. Passerieux, R. Rey, A. Schandrin, M. Urbach, P. Vidailhet, L. Boyer, M. Leboyer. 2017. Relationships between low-grade peripheral inflammation and psychotropic drugs in schizophrenia: results from the national FACE-SZ cohort. European Archives of Psychiatry and Clinical Neuroscience 25. . [Crossref]

3. Jun-Xi Dai, Yan-Bin Ma, Nan-Yang Le, Jun Cao, Yang Wang. 2017. Large animal models of traumatic brain injury. International Journal of Neuroscience 22, 1-12. [Crossref]

4. Vahagn C. Nikolian, Baihong Pan, Tomaz Mesar, Isabel S. Dennahy, Patrick E. Georgoff, Xiuzhen Duan, Baoling Liu, Xizi Wu, Michael J. Duggan, Hasan B. Alam, Yongqing Li. 2017. Lung Protective Effects of Low-Volume Resuscitation and Pharmacologic Treatment of Swine Subjected to Polytrauma and Hemorrhagic Shock. Inflammation 40:4, 1264-1274. [Crossref]

5. Simone E. Dekker, Vahagn C. Nikolian, Martin Sillesen, Ted Bambakidis, Patrick Schober, Hasan B. Alam. 2017. Different resuscitation strategies and novel pharmacologic treatment with valproic acid in traumatic brain injury. Journal of Neuroscience Research 16. . [Crossref]

6. Hasan B. Alam. 2017. Trauma care: Finding a better way. PLOS Medicine 14:7, e1002350. [Crossref] 